\title{
The use of nutritional supplements to induce ketosis and reduce symptoms associated with keto-induction: A narrative review
}

\author{
Cliff J. d C. Harvey ${ }^{\text {Corresp., }}{ }^{1}$, Grant M. Schofield ${ }^{1}{ }^{\text {, Micalla Williden }}{ }^{1}$ \\ ${ }^{1}$ Human Potential Centre, Auckland University of Technology, Auckland, New Zealand \\ Corresponding Author: Cliff J. d C. Harvey \\ Email address: cliff@hpn.ac.nz
}

Background. Adaptation to a ketogenic diet (keto-induction) can cause unpleasant symptoms, and this can reduce tolerability of the diet. Several methods have been suggested as useful for encouraging entry into nutritional ketosis (NK) and reducing symptoms of keto-induction. This paper reviews the scientific literature on the effects of these methods on time-to-NK and on symptoms during the keto-induction phase.

Methods. PubMed, Science Direct, CINAHL, MEDLINE, Alt Health Watch, Food Science Source and EBSCO Psychology and Behavioural Sciences Collection electronic databases were searched online. Various purported ketogenic supplements were searched along with the terms "ketogenic diet", "ketogenic", "ketosis" and ketonaemia (/ ketonemia). Additionally, author names and reference lists were used for further search of the selected papers for related references.

Results. Evidence, from one mouse study, suggests that leucine doesn't significantly increase betahydroxybutyrate (BOHB) but the addition of leucine to a ketogenic diet in humans, while increasing the protein-to-fat ratio of the diet, doesn't reduce ketosis. Animal studies indicate that the short chain fatty acids acetic acid and butyric acid, increase ketone body concentrations. However, only one study has been performed in humans. This demonstrated that butyric acid is more ketogenic than either leucine or an 8-chain monoglyceride. Medium-chain triglycerides (MCTs) increase BOHB in a linear, dose-dependent manner, and promote both ketonaemia and ketogenesis. Exogenous ketones promote ketonaemia but may inhibit ketogenesis.

Conclusions. There is a clear ketogenic effect of supplemental MCTs, however, it is unclear whether they independently improve time to NK and reduce symptoms of keto-induction. There is limited research on the potential for other supplements to improve time to NK and reduce symptoms of keto-induction. Few studies have specifically evaluated symptoms and adverse effects of a ketogenic diet during the induction phase. Those that have typically were not designed to evaluate these variables as primary outcomes, and thus, more research is required to elucidate the role that supplementation might play in encouraging ketogenesis, improve time to NK, and reduce symptoms associated with keto-induction. 
The use of nutritional supplements to induce ketosis and reduce symptoms associated with keto-induction: A narrative review

Cliff J. d C. Harvey, ${ }^{1}$ Grant M. Schofield, ${ }^{1}$ Micalla Williden, ${ }^{1}$

${ }^{1}$ Human Potential Centre, AUT University, Auckland, New Zealand

Corresponding author:

Cliff Harvey, 7 Ascension Place, Rosedale, Auckland 0632, New Zealand. +649 478 3702, cliff@hpn.ac.nz 
1 The use of nutritional supplements to induce ketosis and reduce symptoms associated with

2 keto-induction. A narrative review

3

4 ABSTRACT

5 Background. Adaptation to a ketogenic diet (keto-induction) can cause unpleasant symptoms, and

6 this can reduce tolerability of the diet. Several methods have been suggested as useful for

7 encouraging entry into nutritional ketosis (NK) and reducing symptoms of keto-induction. This

8 paper reviews the scientific literature on the effects of these methods on time-to-NK and on

9 symptoms during the keto-induction phase.

10 Methods. PubMed, Science Direct, CINAHL, MEDLINE, Alt Health Watch, Food Science

11 Source and EBSCO Psychology and Behavioural Sciences Collection electronic databases were

12 searched online. Various purported ketogenic supplements were searched along with the terms

13 "ketogenic diet", "ketogenic", "ketosis" and ketonaemia (/ ketonemia). Additionally, author names

14 and reference lists were used for further search of the selected papers for related references.

15 Results. Evidence, from one mouse study, suggests that leucine doesn't significantly increase beta-

16 hydroxybutyrate (BOHB) but the addition of leucine to a ketogenic diet in humans, while

17 increasing the protein-to-fat ratio of the diet, doesn't reduce ketosis. Animal studies indicate that

18 the short chain fatty acids acetic acid and butyric acid, increase ketone body concentrations.

19 However, only one study has been performed in humans. This demonstrated that butyric acid is

20 more ketogenic than either leucine or an 8-chain monoglyceride. Medium-chain triglycerides

21 (MCTs) increase BOHB in a linear, dose-dependent manner, and promote both ketonaemia and

22 ketogenesis. Exogenous ketones promote ketonaemia but may inhibit ketogenesis. 
23 Conclusions. There is a clear ketogenic effect of supplemental MCTs, however, it is unclear

24 whether they independently improve time to NK and reduce symptoms of keto-induction. There

25 is limited research on the potential for other supplements to improve time to NK and reduce

26 symptoms of keto-induction. Few studies have specifically evaluated symptoms and adverse

27 effects of a ketogenic diet during the induction phase. Those that have typically were not designed

28 to evaluate these variables as primary outcomes, and thus, more research is required to elucidate

29 the role that supplementation might play in encouraging ketogenesis, improve time to NK, and

30 reduce symptoms associated with keto-induction.

\section{Introduction}

32 Very-low-carbohydrate ketogenic diets (VLCKDs) are becoming increasingly popular for 33 mainstream and athletic use for a range of outcomes including weight-loss and maintenance, ${ }^{1}$ 34 improved satiety and a reduction in hunger. ${ }^{2-4}$ The diet also offers specific benefits for health 35 conditions ranging from neurological disorders, obesity, and diabetes and other conditions on the 36 spectrum of metabolic syndrome, and offers potential for the adjunct treatment of various 37 cancers. ${ }^{5-15}$ Ketogenic diets elicit a state of ketosis known as 'nutritional ketosis' (NK), a state of 38 hyperketonaemia distinct from pathological ketosis such as diabetic ketoacidosis (DKA). ${ }^{16}$ Ketosis refers to the production of ketone bodies, derived from fats (and some amino acids) for use as an 40 alternative fuel in times of fasting or drastic carbohydrate restriction. A restriction of carbohydrate, 41 either by fasting or by restricting dietary carbohydrate, results in reduced insulin levels, thereby 42 reducing lipogenesis (the creation of fats) and fat accumulation. When glycogen reserves become 43 insufficient to supply the glucose necessary for normal $\beta$-oxidation of fat, via the provision of oxaloacetate in the Krebs cycle, acetyl-CoA is then used instead in the biosynthesis of ketone bodies via acetoacyl-CoA and $\beta$-hydroxy- $\beta$-methylglutaryl-CoA ${ }^{17}$ to ensure provision of fuel to 
46 the Central Nervous System (CNS), which usually relies on glucose. The process of ketogenesis

47 further allows coenzymes to be freed to ensure continued fatty-acid $\beta$-oxidation. ${ }^{17}$ To elicit this

48 carbohydrate restriction, while also providing sufficient alternate fuel to ensure sustainability of

49 the diet, i.e. in comparison to fasting to achieve ketosis, VLCKDs have been used to encourage

50 ketosis. Early research on KDs focussed on children with epilepsy and for this purpose, a VLCKD

51 typically consists of a 3:1 to $4: 1$ ratio of lipid to non-lipid. This treatment for epilepsy was

52 pioneered at Johns Hopkins University Hospital, ${ }^{18,19}$ and is referred to as a 'classic' or 'standard'

53 ketogenic diet.

54 Ketogenic diets are now commonly applied, for a range of desired outcomes, and with differing

55 definitions of what constitutes a ketogenic diet. Both low-energy diets and VLCKDs with fewer

56 than $50 \mathrm{~g}$ of carbohydrate per day typically result in BOHB levels of $\geq 0.5 \mathrm{mmol} . \mathrm{L}^{-1} .^{20}$ This

57 threshold has been used as a cut-off point for entry into ketosis by Guerci and colleagues, ${ }^{21}$ and is

58 commonly applied as a marker for entry into NK in the nutrition field, as compared to the typically

59 higher levels expected in the medical field to elicit beneficial effects for seizure control in epileptic

60 children. $^{22}$

\section{Time to ketosis}

62 There is a paucity of research that identifies specific time points to the now-common definition of

$63 \mathrm{NK}$, as defined by BOHB levels of $\geq 0.5 \mathrm{mmol}^{-1} \mathrm{~L}^{-20,21}$ In a study comparing fasted ketogenic

64 protocols to a more gradual initiation of a ketogenic diet, Bergqvist and colleagues observed that

65 participants fasting, achieved mean levels of $\geq 0.5 \mathrm{mmol} . \mathrm{L}^{-1} \mathrm{BOHB}$, on the day following initiation

66 of the diet, whereas those on a 1:1 ketogenic diet (by weight) achieved the same level two days

67 after initiation of the diet. ${ }^{23}$ Other studies have measured either tangentially or directly, the

68 achievement of 'ketosis' but have not specifically identified the time at which a level of $\geq 0.5$ 
69 mmol.L-1 was achieved. Berry-Kravis and colleagues observed a mean time to ketosis (urinary

$70>80 \mathrm{mg} / \mathrm{dl}$ ) of 42 hours. ${ }^{24}$ Wirrell and colleagues have demonstrated a mean time to ketosis of 33

71 and 58 hours for any trace of urinary ketones or 'good ketosis' (of $>0.8$ mmol.L ${ }^{-1}$ ) respectively. ${ }^{25}$

72 Wusthoff et al. recorded two cases of adults with prolonged nonconvulsive status epilepticus in

73 which 'stable ketosis' was achieved after 8 and 10 days respectively, 3.6 and $>1.6$ mmol.L-1 ${ }^{-26}$ but

74 the definition for ketosis, in this study, was not mentioned and we cannot extrapolate the time to

75 NK as defined in clinical nutrition. Strzelczyk et al. suggested ketosis as the presence of urinary

76 ketones, some 3.5 days after initiation of a ketogenic diet, but at that time participants had achieved

77 serum BOHB of 3.6 mmol.L ${ }^{-1} \cdot{ }^{27}$ Hoorn and colleagues observed no difference between fasted and

78 non-fasted ketogenic protocols for time to ketosis, without specifically describing their definitions

79 for ketosis or the time to ketosis itself. ${ }^{28,29}$

80 So, while the achievement of ketosis has been described in the medical literature, there are

81 inconsistencies in the measurement of, and definition for ketosis in these papers.

82 Adverse effects of keto-induction-the 'keto-flu'

83 Adaptation to a VLCKD, or 'keto-induction', and the achievement of NK, when transitioning from

84 a standard, higher carbohydrate diet, can cause various unpleasant effects. ${ }^{30}$ Symptoms of keto-

85 induction are predominantly constipation, headache, halitosis, muscle cramps, diarrhoea, and

86 general weakness and rash. ${ }^{31,32}$ These occur because of increased urinary sodium, potassium and

87 water loss in response to lowered insulin levels, ${ }^{33-36}$ greatest between days 1-4 of a fast or ketogenic

88 diet, ${ }^{33}$ and transient reductions in glucose provision to the brain, observed to occur on days $1-3$,

89 with blood glucose normalising after day four. ${ }^{37}$ Constipation may result from reduced food

90 volume or reduced fibre intake, although this finding could be due to the groups that have been 
91 studied, which have included children with disabilities, who commonly experience constipation

92 due to immobility. 32

93 These symptoms are often referred to in the mainstream and grey literature as 'keto-flu' but are

94 not well illustrated in the scientific literature. For example, a Google search returns over 22,000

95 results for the term "keto-flu," but the same term searched in MEDLINE Complete, CINAHL

96 Complete, Alt HealthWatch, Food Science Source, SPORT Discus with Full Text, Psychology,

97 and the EBSCO Behavioural Sciences Collection returns no results. Several studies have described

98 adverse effects during ketogenic diets but to our knowledge, no studies have specifically described

99 symptoms of keto-induction in the short time between commencing a ketogenic diet and the

100 achievement of NK.

101 Adverse effects resulting from a VLCKD are likely to reduce compliance and tolerability, ${ }^{38}$ and

102 thus affect the efficacy of these diets as clinical interventions.

103 There have been several methods suggested to reduce symptoms of keto-induction and to reduce

104 the time taken to achieve NK, including the ketogenic amino acid leucine, short chain fatty acids,

105 medium chain fatty acids, and exogenous ketones.

106 The aim of this paper, therefore, is to elucidate the evidence for and against commonly applied

107 nutritional supplements, purported to be ketogenic, to inform clinical practice in the growing field

108 of ketogenic diets for common-use. This paper reviews the available scientific literature relevant

109 to improvements in time to ketosis and symptoms of keto-induction, resulting from these

110 nutritional supplements.

\section{Methods}

112 PubMed, Science Direct, CINAHL, MEDLINE, Alt Health Watch, Food Science Source and

113 EBSCO Psychology and Behavioural Sciences Collection electronic databases were searched 
114 online. Various purported ketogenic supplements, arising from a qualitative appraisal of forums,

115 social media, message boards, and Google searches for ketogenic supplements, were searched

116 along with the terms "ketogenic diet", "ketogenic", "ketosis" and ketonaemia (/ ketonemia).

117 Additionally, author names and reference lists were used for further search of the selected papers

118 for related references. There is a paucity of studies on time to NK and mitigation of symptoms of

119 keto-induction an as data related to the effects of various supplements on time to induction of

120 ketosis and on symptoms of keto-induction are limited, and there is a lack of homogeneity between

121 study objectives, outcomes, and measures, a narrative review style was chosen.

\section{Results}

\section{Leucine}

124 Leucine and lysine are solely ketogenic branched-chain amino acids (BCAAs). Thus, they do not 125 contribute to gluconeogenesis. Higher leucine (and isoleucine) concentrations result from a 126 ketogenic diet and are related to reduced glutamate-to-GABA ratio and this might explain some of 127 the anti-seizure activity of a ketogenic diet in epilepsy. ${ }^{39}$ There appears to be a high affinity of

128 kidney cells for ketogenesis from leucine. ${ }^{40}$

129 Progression of fasting increases the conversion of leucine to ketone bodies and peripheral tissue is 130 catabolised to provide leucine for ketogenesis. ${ }^{41}$ Leucine can also be degraded in rat astroglial cells

131 to the ketone bodies, including BOHB, and when released by these cells, used by neighbouring 132 neurones as a fuel substrate. ${ }^{42}$ Leucine also results in hepatic ketogenesis. ${ }^{43}$ Studies in mice have 133 shown that while ingested L-leucine can reduce seizure activity similarly to a KD, it does not 134 independently increase blood levels of BOHB. ${ }^{44}$ Evangeliou and colleagues have demonstrated 135 that the addition of $20 \mathrm{~g}$ per day of BCAAs, including $9 \mathrm{~g}$ of leucine, in 17 children with intractable 136 epilepsy, altering the ratio of lipid to protein from 4:1 to around 2.5:, had no effect on ketosis, 
137 along with greater reductions in seizure activity. The authors postulated that this could be due to

138 the ketogenic effect of leucine, but may also result from a greater availability of BCAAs. ${ }^{45}$

\section{Short chain fatty acids}

140 Short-chain fatty acids (SCFAs) have carbon chains between two and five in length. These fatty

141 acids include acetic acid (C:2), propionic acid (C:3), butyric acid (C:4), and valeric acid (C:5).

142 Short chain fatty acids, especially butyric acid, are used extensively as a fuel substrate by intestinal

143 epithelial cells. ${ }^{46}$ It is generally accepted that chain length affects the relative deposition of fatty

144 acids into either lymph or the portal vein. ${ }^{47}$ Those short-chain fatty acids that escape metabolism

145 by epithelial cells are, therefore, primarily absorbed via the hepatic portal vein and do not require

146 'bundling' with micelles and chylomicrons for absorption. ${ }^{48}$ The highest quantities of short-chain

147 fatty acids have been observed in portal blood, followed by hepatic, and far less in peripheral

148 blood. ${ }^{49}$ Thus, they bypass the usual route of absorption (for the more common long-chain fatty

149 acids) into the lymphatics and deposition into the bloodstream via the subclavian vein, and instead,

150 are transported via the hepatic portal vein to the liver where they can be converted into the ketone

151 bodies. ${ }^{50-52}$

\section{Acetic acid}

153 Acetic acid is a two-carbon SCFA. It comprises approximately 4-20\% of vinegar. Vinegar has

154 been demonstrated to improve postprandial insulin sensitivity in healthy and diabetic people and 155 improve glycaemic responses to meals. ${ }^{53-55}$ Urinary excretion of acetone (a ketone body) is

156 increased in phloridzinised dogs and fasting rats after feeding with acetic acid. ${ }^{56}$ Acetone is the 157 spontaneous breakdown product of the ketone bodies acetoacetate and BOHB. Thus, it is likely

158 that acetic acid is ketogenic, and has additional benefits for overall metabolic health, however, no

159 research has been performed on acetic acid and its specific effects on the induction of ketosis or 
160 mitigation of keto-induction symptoms in humans. Interestingly, vinegar is commonly prescribed

161 as a 'free food' in ketogenic diet trials, ${ }^{57-59}$ and may provide an under-recognized stimulus for

162 ketogenesis.

163 Butyric acid

164 Butyric acid (BTA) is a four-carbon, short-chain fatty acid found in the milk of ruminants and

165 present in small amounts in many dairy foods. Most BTA in humans is produced by microbial

166 intestinal fermentation of dietary fibre and resistant starch. Most of the butyric acid produced by

167 this fermentation of starches is absorbed and used directly by colonocytes, with most of the

168 remainder absorbed into the hepatic portal vein, and transported to the liver where it can be

169 converted to ketone bodies. ${ }^{51,52}$ A small amount is absorbed directly from the large colon and

170 enters systemic circulation, to be used directly by peripheral tissue. ${ }^{51}$ Butyrate exerts effects

171 directly on the colonic mucosa, including inhibition of inflammation and carcinogenesis,

172 decreasing oxidative stress, and promotion of satiety. ${ }^{60,61}$ Thus, it serves an important role in

173 preserving the health of the colon, microbiota, and may have other beneficial roles for general and

174 systemic health. Animal studies on the ketogenic potential of butyrate are mixed. For example,

175 silage butyrate content has been shown to provide no significant effect on subclinical ketosis in

176 dairy cows, ${ }^{62}$ however, sub-clinical ketosis is higher in those receiving silage higher in butyrate

177 content. ${ }^{63}$

178 In a recent study in humans, the effect of L-leucine, octanoyl-monoacylglycerol (O-MAG), a

179 monoglyceride consisting of an 8-carbon fatty acid, L-carnitine, and butyric acid on acetoacetate

180 and BOHB were studied. Both $2 \mathrm{~g}$ and $4 \mathrm{~g}$ of butyric acid were demonstrated to be more ketogenic

181 than either $5 \mathrm{~g}$ of leucine, or 5 or $10 \mathrm{~g}$ of O-MAG. ${ }^{64}$

\section{Medium Chain Triglycerides}


183 In medium chain triglycerides (MCTs) two-to-three of the fatty acid chains attached to the glycerol

184 backbone are medium in length. These medium-chain fatty acids (MCFAs) are comprised of a 6-

18512 carbon chain. The MCTs are: caproic (C6), caprylic (C8), capric (C10) and lauric acid (C12). ${ }^{65}$

186 Similar to the short-chain fatty acids and unlike long-chain triglycerides (LCTs), MCTs do not

187 require the actions of bile, nor micellar-chylomicron mediated absorption into the lymphatics and

188 instead are diffused directly into the hepatic portal vein and preferentially converted into bio-

189 available ketone bodies in the liver. Huttenlocher and colleagues first demonstrated that diets

190 containing fewer calories from lipids than a 'classic' ketogenic diet-around 60\%-75\% of

191 calories - can induce NK if they include a high proportion of medium chain triglycerides

192 (MCTs). ${ }^{66}$ A VLCKD with $60 \%$ of energy derived from MCTs, a three-fold greater intake of

193 carbohydrate (18\% vs. $6 \%)$ and a $\sim 50 \%(7 \%$ vs. $10 \%)$ increase in protein compared to a standard

194 ketogenic diet induces NK with no appreciable difference in BOHB levels. ${ }^{67}$

195 Dietary MCTs are also known to promote both ketonaemia and ketogenesis in animals ${ }^{68,69}$ and

196 humans with and without health conditions. ${ }^{70,71}$ MCTs promote ketonaemia and ketogenesis

197 (useful to reduce the risk of night-time hypoglycaemic coma) in those with carnitine

198 palmitoyltransferase deficiency, a rare genetic condition which inhibits the ability to produce

199 ketone bodies from long-chain fatty acids. ${ }^{72,73} \mathrm{MCT}$ s also increase BOHB when calorically dose-

200 matched to either LCTs or carbohydrate in single feeding and non-ketogenic diet studies. ${ }^{74-77}$

201 When fed intravenously, MCTs increase ketogenesis when compared to both structurally similar

202 fats $^{78}$ and LCTs. ${ }^{79}, 80$ However, ketogenesis is reduced by the simultaneous application of 203 glucose ${ }^{81}$ It has been demonstrated by Sandstrom and colleagues that in a hypercaloric diet, there 204 are increased BOHB levels observed with the application of MCTs that aren't seen in a hypocaloric 205 state. ${ }^{82}$ 
206 MCTs increase BOHB in a linear and dose-dependent fashion. For example, when eleven pre-term

207 infants were fed formulas with either $25 \%$ or $50 \%$ of fat calories coming from MCTs for at least

20896 hours $(30 \mathrm{kcal} / \mathrm{ml}$, around $50 \%$ calories from fat in total, $10 \%$ protein, $40 \%$ carbohydrate) the

$20950 \%$ MCT formula resulted in a mean plasma level of BOHB of $0.14 \pm 0.03 \mathrm{mmol} / \mathrm{L} /$, a nearly

210 three-fold increase over the lower MCT formula $(0.06 \pm 0.01) .{ }^{83}$

211 While there is a paucity of research on the effect of MCTs on the time taken to achieve NK, MCTs

212 are demonstrably ketogenic and thus, allow induction of NK with lower proportions of fat in the

213 diet, than that used in 'classic' 3 or 4:1 lipid to non-lipid (or 'ketogenic ratio') protocols. When

214 'classic' ketogenic diets with a greater than 3:1 ratio of lipid to non-lipid are compared to MCT

215 ketogenic diets with $60 \%$ of calories from MCT, NK can be achieved with a lower lipid intake.

216 Huttenlocher first observed higher BOHB levels in children with epilepsy aged 2-9 years, at up to

217 one month on an MCT ketogenic diet, and marginally lower after this time, when compared to a

218 classic ketogenic diet, although these differences were not significant ${ }^{84}$ In a study of 55 children

219 with severe epilepsy, Schwartz and colleagues found modified ketogenic diets, MCT ketogenic

220 diets, and classic ketogenic diets to all be 'ketogenic' (inducing NK) with peak ketone body

221 concentrations of approximately $1 \mathrm{mmol} / \mathrm{L}, 1.5 \mathrm{mmol} / \mathrm{L}$ and $4 \mathrm{mmol} / \mathrm{L}$ respectively, after three

222 weeks on the differing ketogenic protocols. ${ }^{85}$ Nine children were subsequently trialled on a second

223 diet and profiled three weeks later. Cumulative results over 24 hours of metabolic testing

224 demonstrate that expression of ketone bodies rises (in order) from a normal diet (little change) to

225 a modified MCT diet, an MCT ketogenic diet, and the greatest rise in ketone bodies over 24 hours

226 resulting from a classic (4:1) ketogenic diet. In a 12-month study, a classic ketogenic diet resulted

227 in higher levels of BOHB (and acetoacetate) over all time periods (three, six, and 12 months) but

228 this was only statistically significant at three and six months $(\mathrm{p}<0.001){ }^{86}$ 
229 After ingestion of MCT at a dosage of $30 \mathrm{~g} \mathrm{MCT} / \mathrm{m}^{2}$ body surface area by nine children (in a study

230 of seizure control), BOHB levels rose progressively after administration from a mean of $0.2 \pm 0.1$

$231 \mathrm{mmol} / \mathrm{L}$ after an overnight fast to $1.05 \pm 0.3 \mathrm{mmol} / \mathrm{L}$ at 180 minutes. Participants reached NK on

232 average at 30-60 min with most participants in NK by the $90^{\text {th }}$ minute, but there was significant

233 variation in BOHB between individuals. ${ }^{87}$ With a lower dosage of $7.5 \mathrm{~g}$ of MCT taken three times

234 per day after an acclimation period of $5 \mathrm{~g}$ MCT taken three times per day for one week, plasma

235 BOHB was higher, yet not inducing NK. ${ }^{88}$

236 Exogenous ketones

237 Exogenous ketone supplements provide BOHB directly to the body without requiring ketogenesis 238 and without concurrent elevations in free fatty acids. ${ }^{89}$ They are considered to be a safe and 239 effective way to increase ketone body concentrations. ${ }^{90}$ Ketone supplements demonstrate promise 240 as potential adjunct treatments for brain injury, ${ }^{91}$ cancer, ${ }^{92,}{ }^{93}$ Angelman syndrome, ${ }^{94}$ for reducing

241 inflammation by suppressing activation of the NLRP3 inflammasome, ${ }^{95}$ and Alzheimer's

242 disease. ${ }^{96}$ Ketone supplements might also improve fueling during exercise, reduce lactate 243 production, and improve performance due to glucose sparing, ${ }^{97}$ and have positive effects on 244 anxiety, ${ }^{96}$ and mental performance and memory. ${ }^{96}$

245 Exogenous ketone supplements are available as either salts or esters of BOHB. Supplements 246 containing ketone salts (KS) are some combination of sodium-, magnesium-, calcium or 247 potassium-BOHB, and are available commercially from several companies under patent. ${ }^{98}$ Ketone 248 esters (KEs) at the time of writing, are only available for research, primarily as 1,3-butanediol 249 monoester of $\mathrm{BOHB}^{90}$ and thus, the animal and human research has mostly focused on the use of 250 ketone esters. Both ketone esters and salts elevate BOHB to levels consistent with $\mathrm{NK},{ }^{99}$ with 251 ketone esters having greater effects on ketonaemia with ketone salts providing significantly higher 
252 reporting of gastrointestinal symptoms. ${ }^{100}$ Ketone salts might provide a greater potential for long-

253 term side effects if the inorganic ion load delivered is excessive for the individual. ${ }^{100}$ Conversely,

254 R-1,3-butanediol from ketone monoesters is readily metabolized in the liver to AcAc. ${ }^{101}$ Clarke et

255 al. detected no R-1,3-butanediol in the plasma of participants taking a ketone monoester 256 supplement, except at the highest dosage of $714 \mathrm{mg} / \mathrm{kg}$ body weight, at which dose plasma R-1,3-

257 butanediol was detectable at a level of $\leq 1.0 \mathrm{mmol} / \mathrm{L}$ and was undetectable 4 hours later. ${ }^{101}$

258 At a dosage of $395 \mathrm{mg} / \mathrm{kg}$ bodyweight, $\mathrm{KE}$ increased BOHB in healthy volunteers from 0.2 $259 \mathrm{mmol} / \mathrm{L}( \pm 0.02)$ at baseline to $3.3 \mathrm{mmol} / \mathrm{L}( \pm 0.2)$ one hour later, ${ }^{102}$ and from $0.16 \mathrm{mmol} / \mathrm{L}( \pm 0.02)$

260 at baseline to $3.16 \mathrm{mmol} / \mathrm{L}( \pm 0.14) .{ }^{103}$ The same dose has been used to determine the effect on

261 ketonaemia of KE taken with or without a meal. BOHB concentration (one-hour post-KE) was

262 lower in those having taken a meal, but both groups achieved levels of ketonaemia consistent with

$263 \mathrm{NK} ; 2.1 \mathrm{mmol} / \mathrm{L}( \pm 0.2)$ and $3.1 \mathrm{mmol} / \mathrm{L}( \pm 0.1)$ respectively. ${ }^{104} \mathrm{In}$ a study using higher dosages

$264(0.573 \mathrm{~g} / \mathrm{kg} \mathrm{BW})$ in healthy male athletes performing an hour of bicycle exercise at $75 \%$ of 265 maximal exercise intensity BOHB levels rose from 0.1 to $3.4 \mathrm{mmol} / \mathrm{L}(\mathrm{p}<0.01)$ following ketone 266 drinks. $^{105}$

267 While it is clear that exogenous ketones increase serum BOHB, they are not ketogenic, and may, 268 in fact, inhibit endogenous ketone production. ${ }^{106}$ In other words, they promote ketonaemia but do 269 not encourage the creation of ketone bodies in the liver. So, it is more accurate to say that 270 exogenous ketones mimic the effects, many of which are positive, of NK, rather than inducing it.

\section{Conclusions}

272 It's unclear at this time whether an elevation in ketones over and above NK would mitigate the 273 effects of keto-induction. It has, for example, been observed that mood is improved within the first 
274 two weeks of a diet irrespective of macronutrient composition, ${ }^{107}$ and only one study, to our

275 knowledge, has demonstrated a correlation between ketone levels and memory performance. ${ }^{108}$

276 Except for MCTs, there is limited research on the ketogenic potential of nutritional supplements,

277 especially in human subjects. While the ketogenic amino acid leucine may not independently

278 encourage ketogenesis to levels consistent with NK, more research is required, and the effect on

279 time to NK and symptoms of keto-induction, particularly in a classic KD, are at this stage 280 unknown.

281 Similarly, there is a paucity of research on the short-chain fatty acids and their effects on

282 ketogenesis. Their mode of absorption and metabolism, like that of MCTs, but perhaps even more

283 rapid, hints at a potential role for encouraging ketogenesis, and thus, the potential for improving

284 time to NK and reducing symptoms of keto-induction.

285 There is a considerable amount of research demonstrating that MCTs promote both primary

286 ketonaemia resulting from the conversion of medium chain fatty acids liberated from MCTs into

287 bio-available ketone bodies, and longer-term ketogenesis by facilitating keto-adaptation.

288 Expression of the ketone body BOHB is increased in a linear, dose-dependent manner in response

289 to oral loads of MCT but it is unclear whether MCTs independently improve time to NK. Modified

290 MCT ketogenic diets do not significantly hasten the induction of NK over a classic ketogenic diet

291 with a minimum of three parts lipid to one part non-lipid, but they do allow NK to occur in diets

292 containing greater amounts of non-lipid macronutrients.

293 There has, however, been little research performed on the application of MCTs to classic ketogenic

294 diets and whether, if applied, they would; a) improve time to NK, b) result in significantly higher

295 levels of BOHB, and c) significantly reduce symptoms of keto-induction. It is also unknown if, in 
296 the context of a ketogenic diet, MCTs provide additional benefits, for example for physical and

297 mental performance and mood.

298 Exogenous ketones are unlikely to be ketogenic per se, and may inhibit ketogenesis, however, the

299 rapid and substantial elevation of $\mathrm{BOHB}$ offers potential to mitigate effects of keto-induction, and

300 thus, could play a role in improving adherence to a ketogenic diet. Newport et al. have reported

301 improvements in mood and cognitive performance resulting from ketone ester treatment over 20-

302 months in an Alzheimer's Disease case. In this case, cognitive performance tracked plasma BOHB

303 concentrations. In a direct, dose-matched comparison, Kesl and colleagues evaluated the effects

304 of ketone esters, salts, MCTs, and MCT + KS on blood BOHB in Sprague-Dawley rats at a dose

305 of $5 \mathrm{~g} / \mathrm{kg}$. At 0,30 , and $60 \mathrm{~min}$ and 4, 8, and $12 \mathrm{hrs}$ post administration (by intragastric gavage)

$306 \mathrm{KS}+\mathrm{MCT}$ and MCT supplementation rapidly elevated and sustained significant BOHB elevation

307 compared to control for the duration of the 4-week study. Ketone salts did not significantly elevate

308 BOHB at any time point tested compared to controls. Ketone ester supplements significantly

309 elevated BOHB levels for the duration of the 4-week study. This further demonstrates, albeit, in

310 non-human subjects, the superiority of KE to KS for elevating BOHB, and the utility of MCT for

311 the same purpose, but is likely to limited in applicability to health and performance as we have

312 seen demonstrable increases in BOHB, consistent with NK levels with supplementation of KS in

313 humans. ${ }^{99}, 100$ Research performed on exogenous ketone supplements is, at this time, highly

314 preliminary, and has been predominantly performed using animal subjects. Further clinical

315 research is required to translate the potential benefits seen in these studies, to human models of 316 disease and disorder. 
317 This review was limited by a dearth of studies demonstrating the effect of supplementation on the

318 time taken to achieve ketosis as defined by the lingua franca of NK, $\geq 0.5 \mathrm{mmol}^{-1} \mathrm{~L}^{-1}$ and on

319 symptoms of keto-induction during this time.

320 While studies have described symptoms arising from a ketogenic diet, few studies have 321 specifically evaluated symptoms and adverse effects of a ketogenic diet during the induction phase,

322 and the studies that have been performed typically have not been designed to evaluate these as

323 primary outcomes, and thus, our conclusions are extrapolated from a variety of sources. There is

324 also little consensus on whether greater levels of BOHB (over and above NK threshold) are, in

325 fact, associated with fewer symptoms of 'keto-flu', nor for that matter with improved outcomes

326 but as previously noted, Newport and colleagues have observed a linear correlation between mood

327 and cognition, and BOHB levels. ${ }^{109}$ Adverse effects associated with the induction of NK might

328 cause increased drop-out rates and preclude some of the positive effects for those that would

329 otherwise benefit from a VLCKD. For example, Yancy and colleagues noted an 8\% overall

330 dropout rate due to difficulties adhering to an LCHF diet, with a further 5\% withdrawing from

331 their study due to adverse effects. ${ }^{31}$ High attrition rates due to tolerability and gastrointestinal side

332 effects have also been noted in childhood epilepsy research utilising VLCKDs. ${ }^{7,29}$

333 Preliminary research suggests that increased BOHB levels and a faster time-to-NK might improve

334 the acceptability of the KD and improve compliance rates, but more research is required to

335 understand the role that supplementation could play in encouraging ketogenesis, improving time

336 to NK, reducing symptoms associated with keto-induction, and the effect this might have on

337 improving adherence to, and outcomes from a VLCKD.

339 Acknowledgements 
340 We acknowledge the support of our colleagues at the Human Potential Centre, AUT University,

341 especially Eric Helms and Simon Thornley, who helped with the final editing of this document,

342 and Darrell Bonetti who provided guidance on the direction of the review.

\section{References}

343 1. Bueno NB, de Melo ISV, de Oliveira SL, da Rocha Ataide T. Very-low-carbohydrate

344 ketogenic diet v. low-fat diet for long-term weight loss: a meta-analysis of randomised controlled 345 trials. British Journal of Nutrition. 2013;110(07):1178-87.

346 2. Paoli A, Bosco G, Camporesi E, Mangar D. Ketosis, ketogenic diet and food intake 347 control: a complex relationship. Frontiers in Psychology. 2015;6.

348 3. McClernon FJ, Yancy WS, Jr., Eberstein JA, Atkins RC, Westman EC. The effects 349 of a low-carbohydrate ketogenic diet and a low-fat diet on mood, hunger, and other self-reported 350 symptoms. Obesity (Silver Spring, Md). 2007;15(1):182-7.

351 4. Johnstone AM, Horgan GW, Murison SD, Bremner DM, Lobley GE. Effects of a 352 high-protein ketogenic diet on hunger, appetite, and weight loss in obese men feeding ad libitum. 353 The American Journal of Clinical Nutrition. 2008;87(1):44-55.

$3545 . \quad$ Lefevre F, Aronson N. Ketogenic Diet for the Treatment of Refractory Epilepsy in 355 Children: A Systematic Review of Efficacy. Pediatrics. 2000;105(4):e46.

356 6. Keene DL. A Systematic Review of the Use of the Ketogenic Diet in Childhood 357 Epilepsy. Pediatric Neurology. 2006;35(1):1-5.

$3587 . \quad$ Levy RG, Cooper PN, Giri P, Pulman J. Ketogenic diet and other dietary treatments 359 for epilepsy. The Cochrane Library. 2012.

360 8. Henderson CB, Filloux FM, Alder SC, Lyon JL, Caplin DA. Efficacy of the 361 Ketogenic Diet as a Treatment Option for Epilepsy: Meta-analysis. Journal of Child Neurology. 362 2006;21(3):193-8.

$3639 . \quad$ Neal EG, Chaffe H, Schwartz RH, Lawson MS, Edwards N, Fitzsimmons G, et al.

364 The ketogenic diet for the treatment of childhood epilepsy: a randomised controlled trial. The 365 Lancet Neurology. 2008;7(6):500-6.

366 10. Paoli A, Rubini A, Volek J, Grimaldi K. Beyond weight loss: a review of the 367 therapeutic uses of very-low-carbohydrate (ketogenic) diets. European journal of clinical nutrition. 368 2013;67(8):789-96.

369 11. Sumithran P, Proietto J. Ketogenic diets for weight loss: A review of their principles, 370 safety and efficacy. Obesity Research \& Clinical Practice. 2008;2(1):1-13.

12. Maalouf M, Rho JM, Mattson MP. The neuroprotective properties of calorie restriction, the ketogenic diet, and ketone bodies. Brain research reviews. 2009;59(2):293-315.

373 13. Castro K, Faccioli LS, Baronio D, Gottfried C, Perry IS, dos Santos Riesgo R. Effect

374 of a ketogenic diet on autism spectrum disorder: A systematic review. Research in Autism 375 Spectrum Disorders. 2015;20:31-8.

376 14. Varshneya K, Carico C, Ortega A, Patil CG. The Efficacy of Ketogenic Diet and 377 Associated Hypoglycemia as an Adjuvant Therapy for High-Grade Gliomas: A Review of the 378 Literature. Cureus. 2015;7(2):e251. 
$37915 . \quad$ Kulak D, Polotsky AJ. Should the ketogenic diet be considered for enhancing 380 fertility? Maturitas. 2013;74(1):10-3.

381 16. Krebs HA. The regulation of the release of ketone bodies by the liver. Advances in 382 Enzyme Regulation. 1966;4(0):339-53.

383 17. Nelson DL, Lehninger AL, Cox MM. Lehninger principles of biochemistry: 384 Macmillan; 2008.

385 18. Livingstone S. Comprehensive Management of Epilepsy in Infancy, Childhood and 386 Adolescence. Archives of Disease in Childhood. 1972;47(255):842-.

387 19. Livingston S, Pauli LL, Pruce I. KETOGENIC DIET IN TREATMENT OF 388 CHILDHOOD EPILEPSY. Developmental Medicine and Child Neurology. 1977;19(6):833-4.

$38920 . \quad$ Gibson A, Seimon R, Lee C, Ayre J, Franklin J, Markovic T, et al. Do ketogenic 390 diets really suppress appetite? A systematic review and meta-analysis. Obesity Reviews. $391 \quad 2015 ; 16(1): 64-76$.

392 21. Guerci B, Benichou M, Floriot M, Bohme P, Fougnot S, Franck P, et al. Accuracy 393 of an Electrochemical Sensor for Measuring Capillary Blood Ketones by Fingerstick Samples During Metabolic Deterioration After Continuous Subcutaneous Insulin Infusion Interruption in Type 1 Diabetic Patients. Diabetes Care. 2003;26(4):1137-41.

396 22. Gilbert DL, Pyzik PL, Freeman JM. The ketogenic diet: seizure control correlates 397 better with serum beta-hydroxybutyrate than with urine ketones. J Child Neurol. 2000;15(12):78739890.

399 23. Bergqvist AGC, Schall JI, Gallagher PR, Cnaan A, Stallings VA. Fasting versus 400 Gradual Initiation of the Ketogenic Diet: A Prospective, Randomized Clinical Trial of Efficacy. 401 Epilepsia. 2005;46(11):1810-9.

402 24. Berry-Kravis E, Booth G, Sanchez AC, Woodbury-Kolb J. Carnitine Levels and the 403 Ketogenic Diet. Epilepsia. 2001;42(11):1445-51.

404 25. Wirrell EC, Darwish HZ, Williams-Dyjur C, Blackman M, Lange V. Is a fast 405 necessary when initiating the ketogenic diet? Journal of child neurology. 2002;17(3):179-82.

$40626 . \quad$ Wusthoff CJ, Kranick SM, Morley JF, Christina Bergqvist AG. The ketogenic diet 407 in treatment of two adults with prolonged nonconvulsive status epilepticus. Epilepsia. 408 2010;51(6):1083-5.

409 27. Strzelczyk A, Reif PS, Bauer S, Belke M, Oertel WH, Knake S, et al. Intravenous 410 initiation and maintenance of ketogenic diet: Proof of concept in super-refractory status 411 epilepticus. Seizure. 2013;22(7):581-3.

412 28. Kang H-C, Lee Y-M, Kim HD, Lee JS, Slama A. Safe and Effective Use of the 413 Ketogenic Diet in Children with Epilepsy and Mitochondrial Respiratory Chain Complex Defects. 414 Epilepsia. 2007;48(1):82-8.

415 29. Chul Kang H, Joo Kim Y, Wook Kim D, Dong Kim H. Efficacy and Safety of the 416 Ketogenic Diet for Intractable Childhood Epilepsy: Korean Multicentric Experience. Epilepsia. 417 2005;46(2):272-9.

$41830 . \quad$ Hartman AL, Vining EP. Clinical aspects of the ketogenic diet. Epilepsia. $419 \quad 2007 ; 48(1): 31-42$.

420 31. Yancy WS, Jr., Olsen MK, Guyton JR, Bakst RP, Westman EC. A Low421 Carbohydrate, Ketogenic Diet versus a Low-Fat Diet To Treat Obesity and Hyperlipidemia: A 422

423 Randomized, Controlled Trial. Annals of Internal Medicine. 2004;140(10):769-77.

32. Kang HC, Chung DE, Kim DW, Kim HD. Early- and Late-onset Complications of 424 the Ketogenic Diet for Intractable Epilepsy. Epilepsia. 2004;45(9):1116-23. 
425 33. Hamwi GJ, Mitchell MC, Wieland RG, Kruger FA, Schachner SS. Sodium and 426 Potassium Metabolism during Starvation. The American Journal of Clinical Nutrition. 427 1967;20(8):897-902.

428 34. DeFronzo RA, Goldberg M, AGus ZS. The effects of glucose and insulin on renal 429 electrolyte transport. Journal of Clinical Investigation. 1976;58(1):83.

$43035 . \quad$ DeFronzo RA. The effect of insulin on renal sodium metabolism. Diabetologia. $431 \quad 1981 ; 21(3): 165-71$.

$43236 . \quad$ Tiwari S, Riazi S, Ecelbarger CA. Insulin's impact on renal sodium transport and 433 blood pressure in health, obesity, and diabetes. American journal of physiology Renal physiology. 434 2007;293(4):F974-84.

435 37. Harber MP, Schenk S, Barkan AL, Horowitz JF. Alterations in carbohydrate 436 metabolism in response to short-term dietary carbohydrate restriction. American Journal of 437 Physiology - Endocrinology and Metabolism. 2005;289(2):E306-E12.

438 38. Vining EP, Freeman JM, Ballaban-Gil K, Camfield CS, Camfield PR, Holmes GL, 439 et al. A multicenter study of the efficacy of the ketogenic diet. Archives of neurology. 440 1998;55(11):1433-7.

441 39. Roy M, Beauvieux M-C, Naulin J, El Hamrani D, Gallis J-L, Cunnane SC, et al. 442 Rapid adaptation of rat brain and liver metabolism to a ketogenic diet: an integrated study using 443 (1)H- and (13)C-NMR spectroscopy. Journal Of Cerebral Blood Flow And Metabolism: Official 444 Journal Of The International Society Of Cerebral Blood Flow And Metabolism. 2015;35(7):115444562.

$44640 . \quad$ Noda C, Ichihara A. Control of ketogenesis from amino acids. IV. Tissue specificity 447 in oxidation of leucine, tyrosine, and lysine. Journal Of Biochemistry. 1976;80(5):1159-64.

448 41. Kulaylat MN, Frexes-Steed M, Geer R, Williams PE, Abumrad NN. The role of 449 leucine in hepatic ketogenesis. Surgery. 1988;103(3):351-60.

450 42. Bixel MG, Hamprecht B. Generation of ketone bodies from leucine by cultured 451 astroglial cells. J Neurochem. 1995;65(6):2450-61.

452 43. Holecek M, Safránek R, Rysavá R, Kadlcíková J, Sprongl L. Acute effects of 453 acidosis on protein and amino acid metabolism in perfused rat liver. International Journal Of 454 Experimental Pathology. 2003;84(4):185-90.

455 44. Hartman AL, Santos P, O’Riordan KJ, Stafstrom CE, Hardwick JM. Potent anti456 seizure effects of D-leucine. Neurobiology of disease. 2015;82:46-53.

457 45. Athanasios E, Martha S, Vai D, Panagiota K, Anestis I, Astrinia S, et al. Branched 458 Chain Amino Acids as Adjunctive Therapy to Ketogenic Diet in Epilepsy: Pilot Study and 459 Hypothesis. Journal of Child Neurology. 2009;24(10):1268-72.

$46046 . \quad$ Wong JMW, de Souza R, Kendall CWC, Emam A, Jenkins DJA. Colonic Health: 461 Fermentation and Short Chain Fatty Acids. Journal of Clinical Gastroenterology. 2006;40(3):23546243.

463 47. $\mathrm{Mu} \mathrm{H}$, Høy C-E. The digestion of dietary triacylglycerols. Progress in Lipid 464 Research. 2004;43(2):105-33.

465 48. Kuksis A. Biochemistry of glycerolipids and formation of chylomicrons. 466 Champaign: AOCS Press; 2000. p. 119-81.

467 49. Cummings JH, Pomare EW, Branch WJ, Naylor CP, Macfarlane GT. Short chain 468 fatty acids in human large intestine, portal, hepatic and venous blood. Gut. 1987;28(10):1221-7. 
$46950 . \quad$ Bugaut M. Occurrence, absorption and metabolism of short chain fatty acids in the 470 digestive tract of mammals. Comparative Biochemistry and Physiology Part B: Comparative 471 Biochemistry. 1987;86(3):439-72.

472 51. Bourassa MW, Alim I, Bultman SJ, Ratan RR. Butyrate, neuroepigenetics and the 473 gut microbiome: Can a high fiber diet improve brain health? Neuroscience Letters. 2016;625:5647463.

475 52. Stilling RM, van de Wouw M, Clarke G, Stanton C, Dinan TG, Cryan JF. The 476 neuropharmacology of butyrate: The bread and butter of the microbiota-gut-brain axis? 477 Neurochemistry International. 2016;99:110-32.

478 53. Johnston CS, Kim CM, Buller AJ. Vinegar improves insulin sensitivity to a high479 carbohydrate meal in subjects with insulin resistance or type 2 diabetes. Diabetes Care. $480 \quad 2004 ; 27(1): 281-2$.

481 54. Liljeberg H, Björck I. Delayed gastric emptying rate may explain improved 482 glycaemia in healthy subjects to a starchy meal with added vinegar. European journal of clinical 483 nutrition. 1998;52(5):368-71.

484 55. Brighenti F, Castellani G, Benini L, Casiraghi MC, Leopardi E, Crovetti R, et al. 485 Effect of neutralized and native vinegar on blood glucose and acetate responses to a mixed meal 486 in healthy subjects. European journal of clinical nutrition. 1995;49(4):242-7.

487 56. MacKay EM, Barnes RH, Carne HO, Wick AN. Ketogenic activity of acetic acid. 488 Journal of Biological Chemistry. 1940;135:157-63.

$48957 . \quad$ Rother ET. Revisão sistemática X revisão narrativa. Acta Paulista de Enfermagem. 490 2007;20:v-vi.

491 58. Perez-Guisado J, Munoz-Serrano A. A pilot study of the Spanish Ketogenic 492 Mediterranean Diet: an effective therapy for the metabolic syndrome. Journal of medicinal food. 493 2011;14(7-8):681-7.

$49459 . \quad$ Nebeling LC, Lerner E. Implementing A Ketogenic Diet Based on Medium-chain 495 Triglyceride Oil in Pediatric Patients with Cancer. Journal of the American Dietetic Association. 496 1995;95(6):693-7.

497 60. Hamer HM, Jonkers D, Venema K, Vanhoutvin S, Troost FJ, Brummer RJ. Review 498 article: the role of butyrate on colonic function. Alimentary Pharmacology \& Therapeutics. 499 2008;27(2):104-19.

500 61. Fung KY, Cosgrove L, Lockett T, Head R, Topping DL. A review of the potential 501 mechanisms for the lowering of colorectal oncogenesis by butyrate. British Journal of Nutrition. $502 \quad 2012 ; 108(05): 820-31$.

503 62. Samiei A, Liang J, Ghorbani G, Hirooka H, Ansari-Mahyari S, Sadri H, et al. 504 Relationship between dietary energy level, silage butyric acid and body condition score with 505

506

507

508

509

510

511

512

513 subclinical ketosis incidence in dairy cows. Adv Anim Vet Sci. 2015;3(6):354-61.

63. Vicente F, Rodriguez ML, Martinez-Fernandez A, Soldado A, Argamenteria A, Pelaez M, et al. Subclinical ketosis on dairy cows in transition period in farms with contrasting butyric acid contents in silages. TheScientificWorldJournal. 2014;2014:279614.

64. St-Pierre V, Courchesne-Loyer A, Vandenberghe C, Hennebelle M, Castellano C-A, Cunnane SC. Butyrate is more ketogenic than leucine or octanoate-monoacylglycerol in healthy adult humans. Journal of Functional Foods. 2017;32:170-5.

65. Marten B, Pfeuffer M, Schrezenmeir J. Medium-chain triglycerides. International Dairy Journal. 2006;16(11):1374-82. 
514 66. Huttenlocher P, Wilbourn A, Signore J. Medium-chain triglycerides as a therapy for 515 intractable childhood epilepsy. Neurology. 1971;21(11):1097-.

516 67. Huttenlocher PR. Ketonemia and Seizures: Metabolic and Anticonvulsant Effects of

517 Two Ketogenic Diets in Childhood Epilepsy. Pediatr Res. 1976;10(5):536-40.

518 68. Bach A, Schirardin H, Weryha A, Bauer M. Ketogenic response to medium-chain 519 triglyceride load in the rat. The Journal of nutrition. 1977;107(10):1863-70.

520 69. Yeh YY, Zee P. Relation of ketosis to metabolic changes induced by acute medium521 chain triglyceride feeding in rats. The Journal of nutrition. 1976;106(1):58-67.

522 70. St-Onge M-P, Ross R, Parsons WD, Jones PJH. Medium-Chain Triglycerides 523 Increase Energy Expenditure and Decrease Adiposity in Overweight Men. Obesity Research. 524 2003;11(3):395-402.

525 71. Yajnik CS, Sardesai BS, Bhat DS, Naik SS, Raut KN, Shelgikar KM, et al. Ketosis 526 resistance in fibrocalculous pancreatic diabetes: II. Hepatic ketogenesis after oral medium-chain 527 triglycerides. Metabolism: Clinical And Experimental. 1997;46(1):1-4.

528 72. Bonnefont JP, Haas R, Wolff J, Thuy LP, Buchta R, Carroll JE, et al. Deficiency of 529 carnitine palmitoyltransferase I. Journal Of Child Neurology. 1989;4(3):198-203.

530 73. Bougnères PF, Saudubray JM, Marsac C, Bernard O, Odièvre M, Girard J. Fasting 531 hypoglycemia resulting from hepatic carnitine palmitoyl transferase deficiency. The Journal Of 532 Pediatrics. 1981;98(5):742-6.

533 74. Decombaz J, Arnaud MJ, Milon H, Moesch H, Philippossian G, Thelin AL, et al. 534 Energy metabolism of medium-chain triglycerides versus carbohydrates during exercise. Eur J 535 Appl Physiol Occup Physiol. 1983;52(1):9-14.

536 75. Seaton TB, Welle SL, Warenko MK, Campbell RG. Thermic effect of medium-chain 537 and long-chain triglycerides in man. American Journal of Clinical Nutrition. 1986;44(5):630-4.

$53876 . \quad$ Yost TJ, Eckel RH. Hypocaloric feeding in obese women: metabolic effects of 539 medium-chain triglyceride substitution. American Journal of Clinical Nutrition. 1989;49(2):32654030.

$54177 . \quad$ Krotkiewski M. Value of VLCD supplementation with medium chain triglycerides. 542 Int J Obes Relat Metab Disord. 2001;25(9):1393-400.

543 78. Mingrone G, Greco AV, Castagneto M, De Gaetano A, Tataranni PA, Raguso C. 544 Kinetics and thermogenesis of medium-chain monocarboxylic and dicarboxylic acids in man: 545 sebacate and medium-chain triglycerides. JPEN Journal Of Parenteral And Enteral Nutrition. 546 1993;17(3):257-64.

547 79. Jiang ZM, Zhang SY, Wang XR, Yang NF, Zhu Y, Wilmore D. A comparison of 548 medium-chain and long-chain triglycerides in surgical patients. Annals Of Surgery. $549 \quad 1993 ; 217(2): 175-84$.

550 80. Lai H, Chen W. Effects of medium-chain and long-chain triacylglycerols in pediatric 551 surgical patients. Nutrition. 2000;16(6):401-6.

552 81. Kolb S, Sailer D. Effect of fat emulsions containing medium-chain triglycerides and 553 glucose on ketone body production and excretion. JPEN J Parenter Enteral Nutr. 1984;8(3):285-9. 554 82. Sandström R, Hyltander A, Körner U, Lundholm K. Structured triglycerides were 555 well tolerated and induced increased whole body fat oxidation compared with long-chain 556 triglycerides in postoperative patients. JPEN Journal Of Parenteral And Enteral Nutrition. 557 1995;19(5):381-6. 
558 83. Wu PY, Edmond J, Auestad N, Rambathla S, Benson J, Picone T. Medium-chain 559 triglycerides in infant formulas and their relation to plasma ketone body concentrations. Pediatr 560 Res. 1986;20(4):338-41.

$56184 . \quad$ Huttenlocher PR. Ketonemia and seizures: metabolic and anticonvulsant effects of 562 two ketogenic diets in childhood epilepsy. Pediatr Res. 1976;10(5):536-40.

$563 \quad 85 . \quad$ Schwartz RM, Boyes S, Aynsley-Green A. Metabolic effects of three ketogenic diets 564 in the treatment of severe epilepsy. Dev Med Child Neurol. 1989;31(2):152-60.

565 86. Neal EG, Chaffe H, Schwartz RH, Lawson MS, Edwards N, Fitzsimmons G, et al. A 566 randomized trial of classical and medium-chain triglyceride ketogenic diets in the treatment of childhood epilepsy. Epilepsia. 2009;50(5):1109-17.

87. Ross DL, Swaiman KF, Torres F, Hansen J. Early biochemical and EEG correlates 569 of the ketogenic diet in children with atypical absence epilepsy. Pediatr Neurol. 1985;1(2):104-8.

570

571

572

573

574

575

576

577

578

579

580

581

582

583

584

585

586

587

588

589

590

591

592

593

594

595

596

597

598

599

600

601

602

603

Courchesne-Loyer A, Fortier M, Tremblay-Mercier J, Chouinard-Watkins R, Roy healthy humans: estimated potential contribution to brain energy metabolism. Nutrition. 2013;29(4):635-40.

89. Veech RL. Ketone ester effects on metabolism and transcription. Journal of Lipid Research. 2014;55(10):2004-6.

90. Hashim SA, VanItallie TB. Ketone body therapy: from the ketogenic diet to the oral administration of ketone ester. Journal Of Lipid Research. 2014;55(9):1818-26.

91. White H, Venkatesh B. Clinical review: Ketones and brain injury. Critical Care. 2011;15(2):219-.

92. Poff AM, Ward N, Seyfried TN, Arnold P, D'Agostino DP. Non-Toxic Metabolic Management of Metastatic Cancer in VM Mice: Novel Combination of Ketogenic Diet, Ketone Supplementation, and Hyperbaric Oxygen Therapy. PloS one. 2015;10(6):e0127407-e.

93. Poff AM, Ari C, Arnold P, Seyfried TN, D'Agostino DP. Ketone supplementation decreases tumor cell viability and prolongs survival of mice with metastatic cancer. International Journal Of Cancer. 2014;135(7):1711-20.

94. Ciarlone SL, Grieco JC, D'Agostino DP, Weeber EJ. Ketone ester supplementation attenuates seizure activity, and improves behavior and hippocampal synaptic plasticity in an Angelman syndrome mouse model. Neurobiology Of Disease. 2016.

95. Youm Y-H, Nguyen KY, Grant RW, Goldberg EL, Bodogai M, Kim D, et al. The ketone metabolite $\beta$-hydroxybutyrate blocks NLRP3 inflammasome-mediated inflammatory disease. Nature Medicine. 2015;21(3):263-9.

96. Kashiwaya Y, Bergman C, Lee J-H, Wan R, King MT, Mughal MR, et al. A ketone ester diet exhibits anxiolytic and cognition-sparing properties, and lessens amyloid and tau pathologies in a mouse model of Alzheimer's disease. Neurobiology Of Aging. 2013;34(6):15309.

97. Okuda Y, Kawai K, Ohmori H, Yamashita K. Ketone body utilization and its metabolic effect in resting muscles of normal and streptozotocin-diabetic rats. Endocrinologia Japonica. 1991;38(3):245-51.

98. D'agostino DP, Arnold P, Kesl S. Compositions and methods for producing elevated and sustained ketosis. Google Patents; 2015.

99. Holdsworth D, Cox P, Clarke K. Oral ketone body supplementation accelerates and enhances glycogen synthesis in human skeletal muscle following exhaustive exercise. J Clin Lab Invest. 1967;19(3):218-28. 
604 100. Stubbs B, Evans R, Clarke K, Cox P, editors. Ketone ester drinks increase blood 605 ketone levels more effectively than ketone salt drinks. Proceedings of The Physiological Society; 606 2016: The Physiological Society.

607 101. Clarke K, Tchabanenko K, Pawlosky R, Carter E, Todd King M, Musa-Veloso K, et 608 al. Kinetics, safety and tolerability of (R)-3-hydroxybutyl (R)-3-hydroxybutyrate in healthy adult 609 subjects. Regulatory toxicology and pharmacology : RTP. 2012;63(3):401-8.

610 102. Stubbs B, Willerton K, Clarke K, Cox P, editors. A ketone ester drink alters levels 611 of circulating lipids and glucose. Proceedings of The Physiological Society; 2015: The 612 Physiological Society.

613 103. Stubbs B, Willerton K, Clarke K, Cox P, editors. A ketone ester drink reduces 614 appetite compared to an isocaloric carbohydrate drink. Proceedings of The Physiological Society; 615 2015: The Physiological Society.

616 104. Stubbs B, Willerton K, Hiyama S, Clarke K, Cox P, editors. Concomitant meal 617 ingestion alters levels of circulating ketone bodies following a ketone ester drink. Proceedings of 618 The Physiological Society; 2015: The Physiological Society.

619 105. Cox P, Ashmore T, Griffin J, Murray A, Clarke K, editors. A ketone ester drink 620 sustains exercise performance whilst reducing muscle glycolysis. Proceedings of The 621 Physiological Society; 2015: The Physiological Society.

622 106. Balasse EO, Neef MA. Inhibition of ketogenesis by ketone bodies in fasting humans. 623 Metabolism. 1975;24(9):999-1007.

624 107. Rosen JC, Gross J, Loew D, Sims EA. Mood and appetite during minimal625 carbohydrate and carbohydrate-supplemented hypocaloric diets. The American Journal of Clinical 626 Nutrition. 1985;42(3):371-9.

627 108. Krikorian R, Shidler MD, Dangelo K, Couch SC, Benoit SC, Clegg DJ. Dietary 628 ketosis enhances memory in mild cognitive impairment. Neurobiology of Aging. 629 2012;33(2):425.e19-.e27.

$630109 . \quad$ Newport MT, VanItallie TB, Kashiwaya Y, King MT, Veech RL. A new way to 631 produce hyperketonemia: use of ketone ester in a case of Alzheimer's disease. Alzheimer's \& 632 Dementia: The Journal Of The Alzheimer's Association. 2015;11(1):99-103.

633 